\title{
ANÁLISE DA PNEUMATIZAÇÃO DO SEIO ESFENOIDAL EM RELAÇÃO À
} GLÂNDULA HIPÓFISE

\section{ANALYSIS OF PNEUMATIZATION OF THE SPHENOID SINUS IN RELATION TO THE PITUITARY GLAND}

\author{
Mariana Lafetá Lima \\ Maria José Albuquerque Pereira de Sousa e Tucunduva**
}

\begin{abstract}
RESUMO
Introdução: O seio esfenoidal é uma cavidade encontrada no corpo do osso esfenoide. Seu grau de pneumatização é variável, podendo abranger todo o corpo do osso esfenoide. Essa cavidade está localizada logo abaixo da sela turca, que aloja a glândula hipófise. No caso da instalação de um processo infeccioso no seio (sinusite), apenas uma fina camada de mucosa e cortical óssea separará a afeção da glândula, principal responsável pela produção de hormônios corpóreos, assim o grau de pneumatização pode levar ao adelgaçamento da cortical óssea de modo a predispor ao risco de infecção da glândula. O grau de pneumatização e a presença de afecção podem ser observados pela tomografia computadorizada, sugerindo ao clínico a conduta a ser tomada diante de um possível acometimento das estruturas que circundam o seio esfenoidal como a glândula hipófise. Metodologia: Foram observados 81 exames de tomografias computadorizada helicoidal, de pacientes com solicitação de exame dos seios paranasais e cavidade nasal e foram selecionadas quatro imagens de cada paciente, duas referentes ao corte coronal e duas ao corte axial e instituído um padrão de caracterização do seio esfenoidal. D iscussão e resultado: De acordo com a amostra utilizada, foi possível concluir que o seio esfenoidal apresenta-se pneumatizado até a porção posterior do osso esfenoide de modo que envolve a sela turca e, portanto, se aproxima da glândula da hipófise.
\end{abstract}

DESCRITORES: Seio esfenoidal • Osso esfenoide • Hipófise

\section{ABSTRACT}

Introduction: The sphenoid sinus is a cavity found in the body of the sphenoid bone. The degree of pneumatization of this sinus is variable and may cover the entire body of sphenoid bone. This cavity is located just below the sella turcica, which houses the pituitary gland. When an infectious process is located within the sinus (sinusitis), only a thin layer of mucosa and cortical bone separate the pituitary gland, responsible for production of important hormones. The degree of pneumatization can lead to thinning of the cortical bone and thus increase the risk of infecting the gland. The degree of pneumatization and the presence of disease can be observed by CT scan, suggesting to the clinician what action should be taken against a possible involvement of the structures surrounding the sphenoid sinus and the pituitary gland. Methodology: 81 helical computed tomography scans of patients requesting examination of the paranasal sinuses and nasal cavity were observed. Four images were selected for each patient, two coronal and two axial to establish the standard characterization of sphenoid sinus. Result and D iscussion: According to the sample used, it was possible to conclude that the sphenoid presents pneumatized to the posterior portion of the sphenoid bone so that it involves the sella turcica and therefore approaches the pituitary gland.

DESCRIPTORS: Sphenoid sinus • Sphenoid bone • Pituitary gland.

* Discente do Curso de Medicina - UNIVERSIDADE CIDADE DE SÃO PAULO

** Docente do Curso de Medicina - UNIVERSIDADE CIDADE DE SÃO PAULO 


\section{N T R O D UÇÃO}

O seio esfenoidal está situado dentro do corpo do osso esfenoide e é reconhecido como uma cavidade irregular, com o grau de pneumatização variando de ausente a extensa. Normalmente, a pneumatização do seio esfenoidal é descoberta por acaso, por meio de radiografias do crânio, tomografia computadorizada (TC) ou mesmo por meio da radiografia panorâmica. Quando há alguma variação presente, o paciente pode não apresentar qualquer sintomatologia, não sendo necessário tratamento. Dos métodos de imagem com finalidade diagnóstica, a tomografia tem sido mais indicada por promover melhores condições de observação das estruturas nasais e paranasais, uma vez que elimina a sobreposição de imagens. Em um estudo realizado com 109 tomografias computadorizadas, foram encontradas variações no processo pterigoide, sendo $10,1 \%$ do lado esquerdo, $0,9 \%$ do lado direito e $27,5 \%$ em ambos os lados. A pneumatização dessas estruturas ósseas tem importância cirúrgica pelo fato de que estruturas vasculares e nervosas poderiam ser expostas ou até mesmo comprometidas se essa pneumatização não for considerada no processo' ${ }^{1}$.

Além da pneumatização relativa ao corpo do esfenoide, outras variações como a pneumatização do processo pterigoide também podem ser encontradas. A pneumatização do processo pterigoide devido à expansão do seio esfenoide é uma variação anatômica que pode ser negligenciada quando é feito um exame de tomografia computadorizada tendo em vista o portfólio de exames prévios a processos cirúrgicos. Na face posterior da fossa pterigopalatina, encontra-se a face anterior do processo pterigoide do osso esfenoide. Nessa região, está localizada a abertura anterior dos canais redondo e pterigoide, atravessados por veias, artérias e nervos homônimos além dos vasos linfáticos ${ }^{1}$.

A formação do seio esfenoidal é lenta, se inicia no quinto mês pós-natal e seu estado final é atingido com a presença do basiesfenoide, uma porção articular entre a porção basilar do osso occipital com o osso esfenoide que ossifica em função da idade, fenômeno que ocorre no decorrer do sétimo mês de vida. O seio esfenoidal tem origem mais tardia, a partir do recesso esfenoetmoidal².

Há uma semelhança entre o seio esfenoidal e o seio frontal, por apresentarem ossificações bilaterais e um septo ósseo sagital (paramediano), individualizando os dois seios ${ }^{2}$.

O seio esfenoidal tem uma forma de cubo, apresentando quatro paredes, um teto e um assoalho, e apresenta importantes implicações cirúrgicas. A parede anterior é côncava em direção ao seio e apresenta inferior e medialmente o óstio do seio esfenoidal. Essa parede compete com a parede posterior da última célula etmoidal. A parede lateral limita-se em forma ovalada com o seio cavernoso e o dobramento da dura-máter, contendo um parênquima fibroso reticular contendo sangue venoso e atravessada pela artéria carótida interna e nervos cranianos III, IV e VI, tangenciada lateral e superiormente pelo V1 e V2, ambos do nervo trigêmeo, $\checkmark$ par craniano ${ }^{2}$.

A parede posterior do seio tem relação com o processo basilar do corpo do esfenoide. Em sua junção com a parede lateral, na parte mais exterior, localiza-se a artéria carótida interna, em seu trajeto ascendente, ao penetrar no seio cavernoso. A parede medial corresponde ao septo que, em sua maioria, tem deslocamento para a direita. No lado nasal, a convexidade dessa parede articula-se com o vômer, constituindo-se na região coanal superior, transição nasofaríngea. Bilateralmente ao septo vomeriano, na parte mais medial, encontram-se os óstios esfenoidais ${ }^{3}$.

O teto do seio esfenoidal limita-se com a fossa hipofisária, sua expansão superior está quase sempre deslocada anterolateral e superiormente, podendo vir a comprometer o canal óptico. O assoalho do seio esfenoidal tem uma liberdade de expansão, mas esta ocorre, na maioria das vezes, em relação à raiz do processo pterigoide ${ }^{3}$.

Liang et al. ${ }^{4}$ em 2001, fizeram um relato de um caso no qual houve a pneumatização do osso esfenoide, acometendo o processo pterigoideo. Essa variação anatô-
LIMA ML

tucunduva mJaps

ANÁLISE DA

PNEUMATIZAÇÃO

DO SEIO

ESFENOIDAL

EM RELAÇÃO

À GLÂNDULA

HIPÓFISE

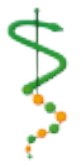

REV, ODONTOL.

UNIV, CID, SÃO

PAULO

$2013 ; 25(3): 216-$

22, SET-DEZ 
LIMA $M L$

TUCUNDUVA MJAPS

ANÁLISE DA PNEUMATIZAÇÃO

DO SEIO

ESFENOIDAL

EM RELAÇÃO

À GLÂNDULA HIPÓFISE

\section{8}

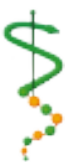

REV, ODONTOL.

UNIV, CID, SÃO

PAULO

$2013 ; 25(3): 216$

22, SET-DEZ
I SSN 1983-5183

mica foi identificada em uma radiografia panorâmica e confirmada em tomografia computadorizada preoperatória, previamente à instalação de implantes dentais.

Dias et al. ${ }^{2}$ (2004) realizaram uma análise do grau de pneumatização do seio esfenoidal, de acordo com a projeção do nervo óptico, e verificaram que o aumento da pneumatização esteve associado ao maior grau de projeção e deiscência do nervo óptico na parede desse seio. Alguns autores Hammer e Radberg 5 (1961), Wigand $^{6}$ (1990), Navarro ${ }^{7}$ (1999) e Terrier ${ }^{8}$ (1991) observaram que a pneumatização do seio esfenoidal aumentava a exposição e vulnerabilidade do nervo óptico.

Dias et al. ${ }^{2}$ (2004) classificaram os tipos de seio esfenoidal em conchal, pré-selar e selar, no qual a pneumatização estendia-se para o corpo do esfenoide e, mais posteriormente, em direção à porção basilar. Já nos estudos realizados por Santos et al. ${ }^{9}$ (2007), quanto mais pneumatizado for o seio esfenoidal, maior o número de variações anatômicas presentes. Em relação a sua pneumatização, $\mathrm{O}$ seio esfenoidal pode ser classificado em três tipos: o tipo conchal, que não atinge o corpo do esfenoide, sendo um seio pequeno, separado da sela túrcica por uma parede óssea espessa; o tipo pré-selar, no qual o limite posterior do seio não ultrapassa a metade anterior do assoalho selar e o tipo selar, sendo este o mais frequente, no qual a cavidade estendia-se por baixo da sela até a porção basilar. Geralmente não era possível a identificação de todas essas variações anatômicas nem mesmo nos seios selares; no entanto, a identificação do plano esfenoidal, da porção basilar e das proeminências ósseas das artérias carótidas internas pode ser considerada suficiente para a determinação dos limites do assoalho selar com segurança. $\mathrm{Na}$ presença de um seio do tipo pré-selar ou conchal, e ausência de referências anatômicas suficientes, foi utilizado o intensificador de imagem para confirmação da trajetória cirúrgica 9 .

Navarro $^{10}$ (1996) descreveu a pneumatização do seio esfenoidal com expansão para o osso occipital em $40 \%$ dos casos. Delano et al. ${ }^{1}$ (1996) encontraram pneumatização do processo clinoide anterior em $4 \%$ dos casos ao avaliar exames de tomografia computadorizada.

White et al. ${ }^{11}$ (2003) relataram que o grau de aeração do seio estava ligado ao início de fístulas liquóricas espontâneas, aumentando a pneumatização do seio esfenoidal, levando a uma maior reabsorção óssea e também a prolapsos da dura- máter.

Araújo Filho 3 (2008) em seus estudos obteve resultados em porcentagens do processo de pneumatização do seio esfenoidal de: $10,1 \%$ à esquerda, $0,9 \%$ à direita e $27,5 \%$ bilateralmente.

Analisando os diversos trabalhos realizados para avaliação do seio esfenoidal, nota-se que a maioria deles foi feita com tomografias computadorizadas, nas quais a imagem criada representa uma parte da estrutura a ser estudada.

\section{MATERIAL E MÉTODOS}

Esta pesquisa foi aprovada pelo Comitê de Ética em Pesquisa (CEP) da Universidade Cidade de São Paulo com o protocolo de pesquisa $n^{\circ} 13668704$, sendo aprovada em 13 de junho de 2012.

Foram observados 81 exames de tomografias computadorizadas helicoidais, pertencentes a um banco de dados, de pacientes com solicitação de exame dos seios paranasais e cavidade nasal.

Inicialmente foram selecionadas quatro imagens de cada paciente, duas referentes ao corte coronal e duas ao corte axial. Após selecionadas as imagens, foi instituído um padrão para comparação e determinação dos limites da pneumatização relacionados à sela turca. Todas as imagens foram analisadas e os resultados encontrados passaram por uma avaliação estatística.

\section{RESULTADOS}

A amostra contou com 81 exames de tomografia computadorizada, composta por 37 homens e 44 mulheres, sendo estabelecidos os seguintes limites para análise, utilizados como padrão em ambos os cortes:

anterior - antes do processo clinoide anterior e tubérculo da sela

limite anterior - exatamente no processo clinoide anterior e tubérculo da sela 
central - no centro da sela turca

limite posterior - exatamente no processo clinoide posterior e dorso da sela

posterior - posterior ao processo clinoide posterior e dorso da sela.

De acordo com a amostra analisada foram encontrados os resultados descritos a seguir.

Quando apenas o corte utilizado foi avaliado, no corte coronal, o padrão mais identificado foi o posterior (E - Fig. 1) com $61,76 \%$, seguido por limite posterior (D Fig. 2) com 14,17\%, central (C - Fig. 3) com 5,6\%, limite anterior (B - Fig. 4) com $1,1 \%$, e anterior (A) $0,0 \%$. Levando-se em consideração o corte axial, o padrão mais identificado foi o limite posterior (D - Fig. 5) com $44,54 \%$, seguido por limite anterior (B - Fig. 6) com 13,16\%, central (C - Fig. 7) com 11,14\%, posterior (E - Fig. 8) com $8,10 \%$ e anterior (A - Fig. 9) com $5,6 \%$. Comparando os resultados obtidos em ambos os cortes, os mesmos foram diferentes, observou-se que no corte coronal o padrão mais observado foi o posterior ( $E$ - Fig. 1), já no corte axial o padrão mais identificado foi o limite posterior (D - Fig. 5). Outra diferença que se pode observar é que no corte coronal não foi identificado nenhum resultado no padrão anterior $(\mathrm{A})$; já no corte axial, o padrão anterior (A Fig. 9) foi identificado, ainda que em menor quantidade quando comparado aos outros padrões.

De acordo com a classificação utilizada no presente trabalho, e considerando ambos os cortes, coronal e axial, os padrões mais observados nas imagens foram

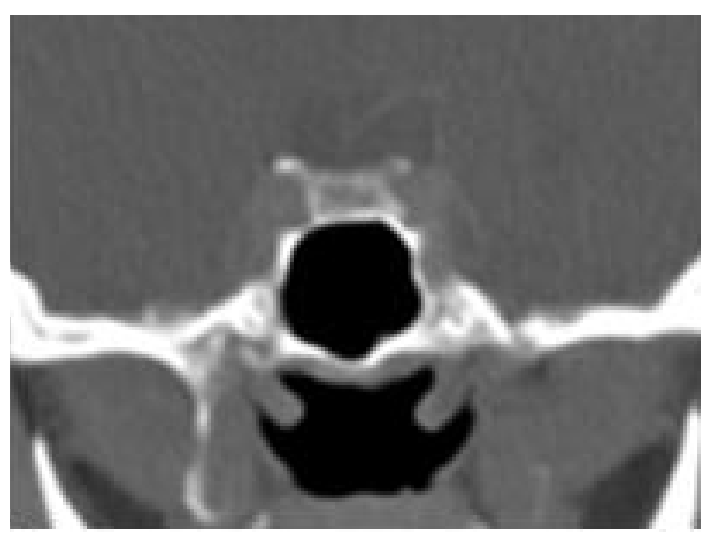

Fig. 1 - Imagem de TC em corte coronal, apresentando pneumatização do seio esfenoidal posterior ao processo clinóide posterior e dorso de sela (E). os de limite posterior (D - Fig. 2 e Fig. 5) e posterior (E - Fig. 1 e Fig. 8).

Quando considerada a idade, independente de gênero, no corte axial o padrão mais identificado foi também o limite posterior (D). Já no corte coronal o padrão mais identificado foi posterior (E). Quando se avaliou idade e mulher, o padrão mais identificado em corte axial foi limite posterior (D) e, no corte coronal, o padrão posterior (E). Avaliando-se idade e homem, o padrão mais identificado em corte axial foi limite posterior (D) e, no corte coronal, o padrão posterior (E). Assim, pode-se concluir que, independente do sexo, o resultado encontrado em relação à pneumatização do seio foi o mesmo.

\section{I SCUSSÃO}

De acordo com Navarro7 (1999), o seio esfenoidal tem 4 paredes, um teto e um assoalho. O teto foi o que apresentou relação com a análise realizada neste trabaIho, pois limita-se com a fossa hipofisária. A análise da pneumatização do seio em muitos trabalhos, como os de Navarro ${ }^{7,10}$ (1996 e 1999), Dias et al. ${ }^{2}$ (2004), Hammer e Radberg ${ }^{5}$ (1961), Wigand ${ }^{6}$ (1990) e Terrier $^{8}$ (1991) foi estudada relacionando-se ao nervo óptico e canal óptico, mas nada foi citado em relação à localização ou o acometimento da glândula hipófise.

O presente trabalho classificou a pneumatização do seio esfenoidal em: anterior (A), limite anterior (B), central (C), limite posterior (D) e posterior (E), classificação

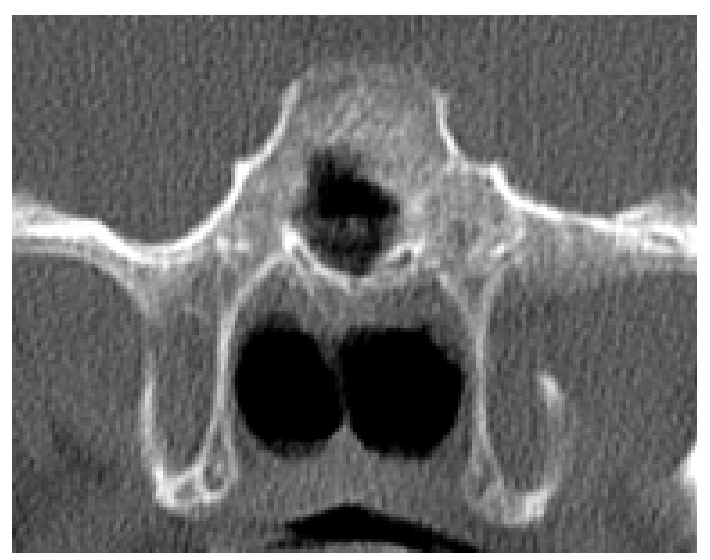

Fig. 2 - Imagem de TC em corte coronal, apresentando pneumatização do seio esfenoidal exatamente no processo clinóide posterior e dorso de sela (D).
LIMA ML

TUCUNDUVA MJAPS

ANÁLISE DA

PNEUMATIZAÇÃO

DO SEIO

ESFENOIDAL

EM RELAÇÃO

À GLÂNDULA

HIPÓFISE

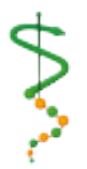

REV, ODONTOL.

UNIV, CID, SÃO

PAULO

2013; 25(3): 216 -

22, SET-DEZ 
LIMA $M L$ TUCUNDUVA MJAPS

ANÁLISE DA PNEUMATIZAÇÃO

DO SEIO

ESFENOIDAL

EM RELAÇÃO

À GLÂNDULA

HIPÓFISE

220

REV. ODONTOL UNIV, CID, SÃO

PAULO

$2013 ; 25(3): 216$

22, SET-DEZ :

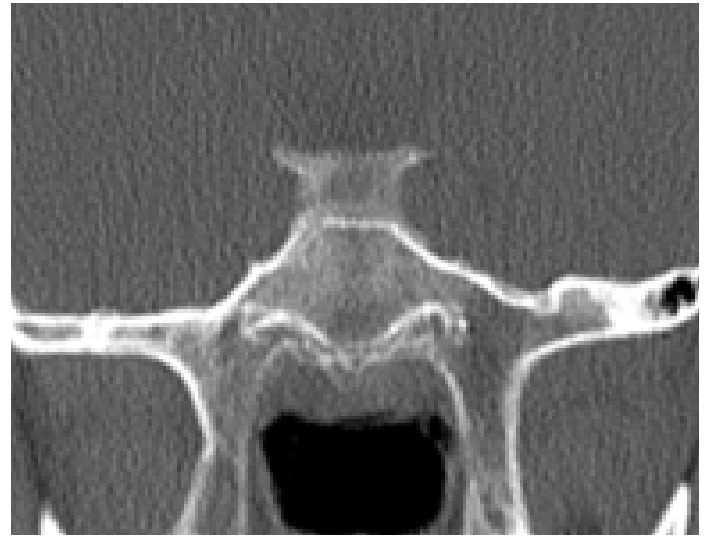

Fig. 3 - Imagem de TC em corte coronal, apresentando pneumatização do seio esfenoidal no centro da sela Turca (C).

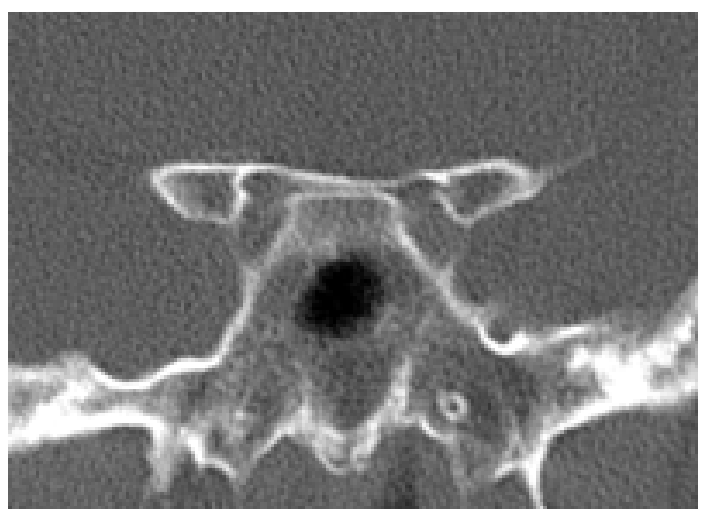

Fig. 4 - Imagem de TC em corte coronal, apresentando pneumatização do seio esfenoidal exatamente no processo clinóide anterior e tubérculo da sela (B).

diferente da encontrada na literatura no trabalho de Dias et al. ${ }^{2}$ (2004), que classificaram os tipos de seio esfenoidal em conchal, pré-selar e selar. Comparando-se os padrões de ambos os trabalhos, pode-se concluir que o tipo conchal seria o mesmo do tipo anterior $(\mathrm{A})$, o tipo pré-selar estaria abrangendo o tipo limite anterior (B) e central (C) e, por fim, o tipo selar estaria relacionado ao padrão limite posterior (D) e posterior (E). O tipo selar foi o mais frequente no trabalho de Dias et al. ${ }^{2}$ (2004), assim como no presente trabaIho, em que os padrões de limite posterior (D) e posterior (E) também foram os mais observados.

Durante o estudo, houve um trabalho de Araújo Filho $^{3}$ (2008) que observou, além da pneumatização do seio esfenoidal, variações na pneumatização do processo pterigoide, o que não foi avaliado

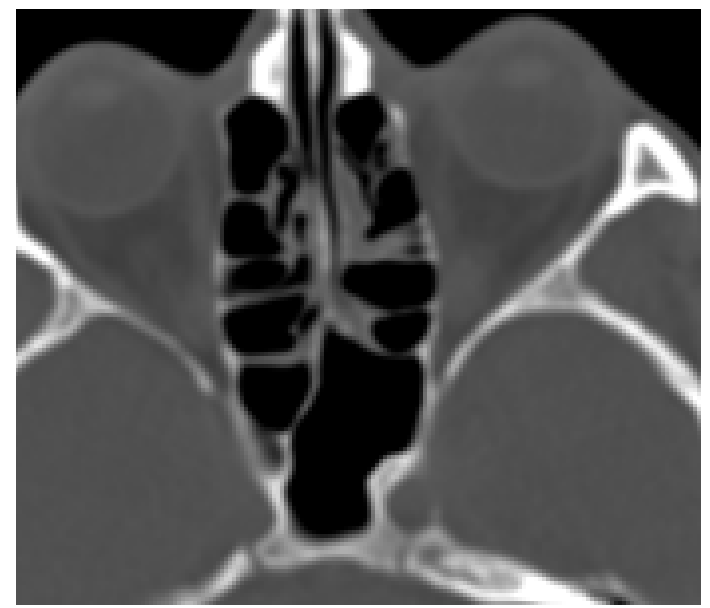

Fig. 5 - Imagem de TC em corte axial, apresentando pneumatização do seio esfenoidal exatamente no processo clinóide posterior e dorso da sela (D).

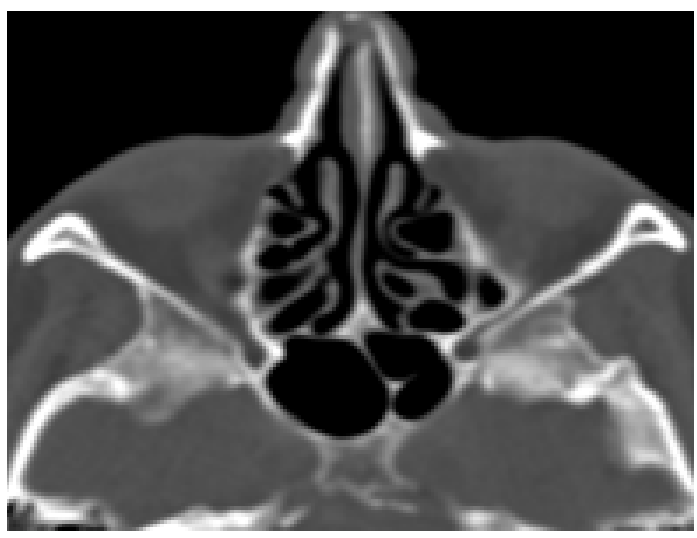

Fig. 6 - Imagem de TC em corte axial, apresentando pneumatização do seio esfenoidal até exatamente no processo clinóide anterior e tubérculo da sela $(B)$.

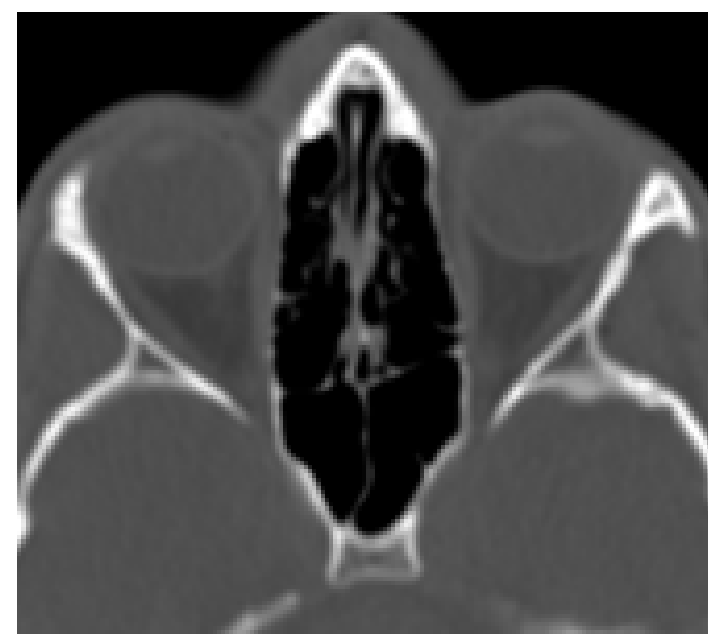

Fig. 7 - Imagem de TC em corte axial, apresentando pneumatização do seio esfenoidal no centro da sela turca (C). 


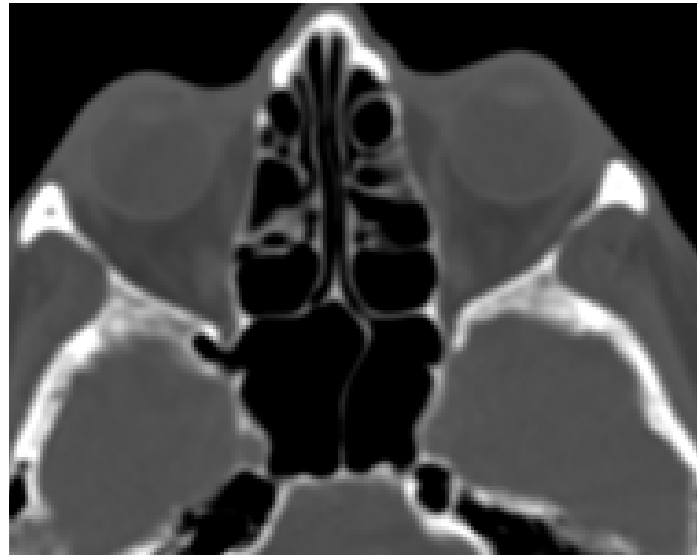

Fig. 8 - Imagem de TC em corte axial, apresentando pneumatização do seio esfenoidal posterior ao processo clinóide posterior e dorso da sela (E).

neste trabalho.

Quando analisados os principais trabalhos que avaliam a pneumatização do seio esfenoidal, o principal exame utilizado foi a tomografia computadorizada, exame utilizado neste trabalho, pois diminuiu a sobreposição de imagens. O presente trabalho avaliou a pneumatização do seio esfenoidal de pacientes que foram encaminhados para avaliação de seios paranasais. Observou-se que a pneumatização do seio, na maioria das vezes, é bem abrangente, podendo englobar toda a base da sela turca, fator às vezes negligenciado, porém de extrema importância durante a análise dos exames, pois o grau de aeração do seio esfenoidal o coloca

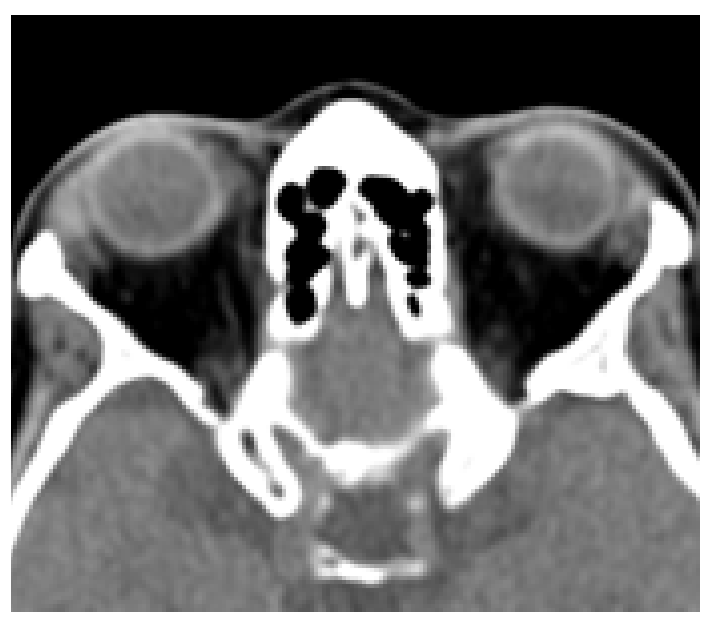

Fig. 9 - Imagem de TC em corte axial, apresentando pneumatização do seio esfenoidal antes do processo clinóide anterior e tubérculo da sela (A).

em maior proximidade à glândula hipófise, assim uma infecção do seio poderia eventualmente acometê-la, assim como foi observado em alguns trabalhos Hammer e Radberg ${ }^{5}$ (1961), Navarro ${ }^{7}$ (1999) e Terrier $^{8}$ (1991) que observaram o mesmo, porém relacionando a pneumatização do seio à vulnerabilidade do nervo óptico.

\section{CONCLUSÃO}

De acordo com a amostra utilizada foi possível concluir que o seio esfenoidal apresenta-se pneumatizado até a porção posterior do osso esfenoide, de modo que envolve a sela turca e, portanto, se aproxima da glândula da hipófise.
LIMA ML

TUCUNDUVA MJAPS

ANÁLISE DA

PNEUMATIZAÇÃO

DO SEIO

ESFENOIDAL

EM RELAÇÃO

À GLÂNDULA

HIPÓFISE

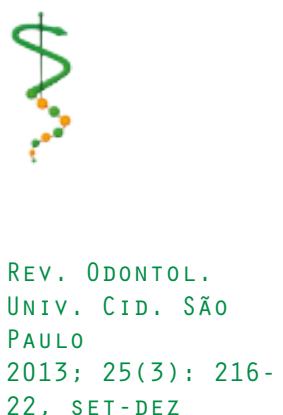


LIMA $M L$

TUCUNDUVA MJAPS

ANÁLISE DA

PNEUMATIZAÇÃO

DO SEIO

ESFENOIDAL

EM RELAÇÃO

À GLÂNDULA HIPÓFISE

\section{2}

1. DeLano MC, Fun FY, Zinreich SJ. Relationship of the optic nerve to the posterior paranasal sinuses: a CT anatomic study. AJNR Am J Neuroradiol 1996 Apr;17(4):669-75.

2. Dias PCJ, Albernaz PLM, Yamashida HK. Relação anatômica do nervo óptico com o seio esfenoidal: estudo por tomografia computadorizada. Rev Bras Otorrinolaringol 2004 out;70(5):6517.

3. Araújo Filho BC. Estudo da anatomia do seio esfenoidal através da dissecção endoscópica em cadáveres. São Paulo: Faculdade de Medicina; 2008.

4. Liang H, Benson BW, Frederiksen NL. Pneumatization of the pterygoid process of the sphenoid bone. Dentomaxillofac Radiol 2001 Jan;30(1):63.

5. Hammer G, Radberg C. The sphenoidal sinus. An anatomical and roentgenologic study with reference to transsphenoid hypophysectomy. Acta radiol 1961 Dec;56(401-22.

6. Wigand EM. Endoscopic surgery of the paranasal sinuses and anterior skull base. New York: Stuttgart; 1990.
7. Navarro JAC, Andreo JC, Navarro PL, Navarro RL. Estudo anatômico dos forames redondo e pterigóide, na fossa pterigopalatina. Rev bras otorrinolaringol 1999 65(9):484-9.

8. Terrier G, Stell PM. Rhinosinusal endoscopy: diagnosis and surgery. Milano: Zambon Group; 1991.

9. Santos RP, Zymberg ST, Abucham Filho JZ, Gregório LC, Weckx LLM. Acesso endoscópico transnasal aos tumores selares. Rev Bras Otorrinolaringol 2007 ago;73(4):463-75.

10. Navarro JAC. Cavidade do nariz e seios paranasais: anatomia cirúrgica. Bauru; All Dent 199663.

11. White DR, Dubin MG, Senior BA. Endoscopic repair of cerebrospinal fluid leaks after neurosurgical procedures. Am J Otolaryngol 2003 Jul-Aug;24(4):213-6.

Recebido em 28-11-2013

Aceito em 04-12-2013 